\title{
Jeanne Louise Poulain d'Andecy, épouse Poincaré (1857-1934)
}

Laurent Rollet ${ }^{1}$

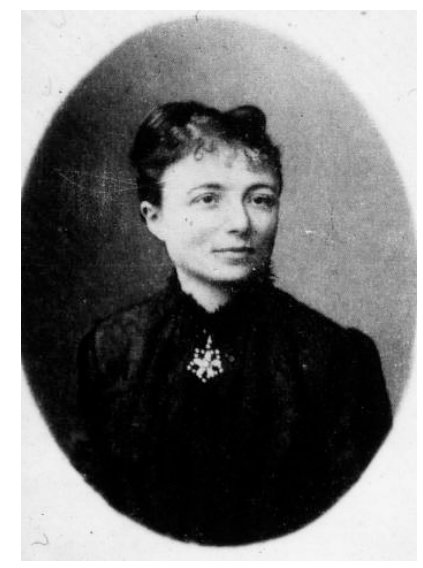

Louise Poincaré vers 30 ans

En mars 1881, Henri Poincaré écrit à son ami Elias Kahn (1855-1913) pour lui annoncer son mariage. Tous deux se sont probablement connus au lycée de Nancy au début des années 1870 en classe de mathématiques spéciales. Ayant été classé premier au concours de l'École polytechnique et cinquième au concours de l'École normale supérieure en 1873, Poincaré avait hésité entre les deux écoles ; cependant, à en croire les souvenirs de sa sœur, il avait finalement choisi de suivre le modèle de son oncle paternel, Antoni Poincaré (1825-1911), et d'opter pour la voie polytechnicienne ${ }^{2}$. Entré avec un rang bien moins prestigieux (248ème sur 261), son ami - qu'il appellera dans d'autres lettres 'Kah-Na-Pesch' ou mon 'frère Topinard' - était sorti de Polytechnique en fin de classement et avait embrassé une carrière dans l'artillerie. Cette annonce de mariage, très courte, est marquée du sceau d'une amitié virile :

Mon cher ami,

Je vais me marier et comme je sais que tu t'intéresses à tout ce qui touche la fouffe ${ }^{3}$ je me hâte de t'annoncer cet événement. J'épouse Mlle d'Andecy qui, j'ai toute raison de le croire, me rendra heureux.

Je te serre affectueusement la main.

Poincaré $^{4}$

\footnotetext{
${ }^{1}$ Université de Lorraine, Laboratoire d'histoire des sciences et de philosophie Archives Henri Poincaré (UMR CNRS 7117), Maison des sciences de l'homme Lorraine (USR 3261).

${ }^{2}$ Boutroux Aline, Vingt ans de ma vie, simple vérité... La jeunesse d'Henri Poincaré racontée par sa sœur (18541878) - Texte inédit édité par Laurent Rollet, Paris, Hermann, 2012, p. 232.

${ }^{3}$ Le terme d'argot " fouffe " désigne le sexe de la femme et, par extension, la femme ou la fille (de manière plutôt péjorative).

${ }^{4}$ Lettre datant probablement de mars 1881, Archives de l'Académie des sciences. Poincaré et Kahn entretiendront une relation amicale durant de nombreuses années, le mathématicien allant même jusqu'à intercéder auprès de son cousin Raymond Poincaré pour lui rendre service à une occasion. Pour la
} 
À cette époque, Henri Poincaré est en poste à la Faculté des sciences de Caen où il est chargé d'un cours de calcul différentiel et intégral. II a soutenu sa thèse en 1879 et il est alors en pleine phase ascendante d'un point de vue scientifique ${ }^{5}$. II aspire manifestement à une carrière à la Sorbonne et il bénéficie de nombreux soutiens, dont celui de son maître Charles Hermite.

Issu de la bourgeoisie intellectuelle nancéienne, Poincaré dispose d'un sérieux réseau de relations familiales, sociales et politiques, au sein duquel on trouve quelques maires, ministres et députés Auguste Bernard (1824-1883), Henri Varroy (1826-1883), Jules Develle (1845-1919) - ainsi des personnalités pour le moins influentes telles que l'historien Alfred Rambaud (1842-1905), un ami très proche de la famille qui sera directeur du cabinet de Jules Ferry au Ministère de I'Instruction publique à partir de 1879, ou le géologue Auguste Daubrée (1814-1896), un cousin de la famille, qui dirigera l'École des mines à partir de 1872 (et donc les études de Poincaré de 1875 à 1878). La lecture de la correspondance de jeunesse du mathématicien ou des souvenirs de sa sœur permet de reconstruire quelques pans de ce réseau de sociabilité et d'ouvrir des perspectives biographiques fécondes.

Pourtant, ces sources ne nous apprennent rien sur Louise Poulain d'Andecy... Où et comment rencontre-t-il celle qui deviendra son épouse ? Comment sont-ils présentés ? Les familles Poincaré et d'Andecy se connaissent-elles de longues date ? S'agit-il d'un mariage d'intérêt ou d'un mariage d'amour ? On sait que dans la famille Poincaré la question des unions d'inclination divise et que la sœur du mathématicien devra quelque peu batailler avec ses proches pour parvenir à évincer les prétendants qui ne lui conviennent pas et épouser le philosophe Émile Boutroux 6 . Mais s'agissant de Poincaré lui-même ces questions restent malheureusement sans réponse.

En attendant la découverte de sources nouvelles, on se contentera ici très modestement d'explorer quelques pistes biographiques sur cette union matrimoniale, qu'il est possible d'attraper par le biais de la figure du beau-père de Poincaré, Jean-Baptiste Henri Poulain d'Andecy. Ce faisant nous montrerons l'importance déterminante des réseaux familiaux pour les débuts de carrière du mathématicien.

\section{Les origines familiales de Louise Poulain d'Andecy (1857- 1934)}

Jeanne Louise Marie Poulain d'Andecy naît à Paris (Passy) le 17 septembre 1859. Elle est la fille de Jean-Baptiste Henri Poulain d'Andecy, qui exerce alors la profession de banquier, et de Pauline Anaïs Louise Geoffroy saint-Hilaire.

Le berceau familial de son père se situe dans les Ardennes. Dans un article sur la famille Poincaré Françoise Poincaré mentionne que dans un premier temps les membres de la famille portaient le nom de Poulain avant d'y accoler le nom du lieu-dit de la propriété familiale située à Andecy, sur la commune de Bouy dans la Marne. Dans la famille Poulain d'Andecy on trouve un député de Vitry-leFrançois ayant refusé de voter la mort de Louis XVI, Jean-Baptiste Célestin (peut-être le grand-père de Louise) ${ }^{7}$. Henri Poulain d'Andecy, le futur beau-père d'Henri Poincaré, voit le jour à Paris le 10 mars 1818. On ne dispose pas d'informations précises sur ses parents, si ce n'est qu'il est issu d'une famille de la haute magistrature parisienne. Il aura deux sœurs nettement plus jeunes que lui dont il devra assumer la charge financière à la mort de ses parents au début des années. Sa famille est sans

correspondance entre les deux hommes, cf. le site web de la correspondance de Poincaré : http://www.univnancy2.fr/poincare/chp/

${ }^{5}$ En 1881, il publie près d'une vingtaine de contributions scientifiques, dont une quinzaine de notes dans les Comptes rendus de l'Académie des sciences (principalement consacrées aux fonctions fuchsiennes).

${ }^{6}$ Boutroux Aline, Vingt ans de ma vie, Op. cit., chapitre XXVIII.

${ }^{7}$ Poincaré Françoise, «La famille Poincaré », Généalogie magazine, 124, 1994, 14-19. 
nul doute très aisée. Elle possède une propriété et des terres dans la région d'Épernay dont la valeur patrimoniale est estimée à 600000 Francs en 1851. Sa condition sociale lui permettra de suivre, comme on le verra, un parcours de vie plutôt libre. II restera célibataire jusqu'à ses 33 ans $^{8}$.

Il épouse le 8 novembre 1851 Pauline Anaïs Louise Geoffroy Saint Hilaire (1831-1885). Treize années les séparent. Son épouse, née le 9 mars 1831 à Paris, est issue d'une prestigieuse famille de naturalistes. Son grand-père, Etienne Geoffroy Saint Hilaire (1772-1844) s'était rendu célèbre pour sa participation à l'expédition scientifique qui avait accompagné Bonaparte en Égypte en 1798. Il y avait mené des études sur les poissons du Nil. A son retour en France il devait entrer à l'Académie des sciences et mener une carrière scientifique marquée par des travaux anatomiques et tératologiques qui s'apparentent au transformisme de Lamarck. Gravement malade à partir de 1840 il avait cédé sa chaire au Muséum d'histoire naturelle à son fils Isidore, né de son mariage avec Pauline Brière de Mondétour (1785-1876), une jeune fille issue d'une famille très influente. Avant d'obtenir cette chaire, Isidore Geoffroy Saint Hilaire (1805-1861), le beau-père d'Henri Poulain d'Andecy, avait mené des travaux en zoologie. Plus tard il devait être nommé professeur à la Faculté des sciences et fonder la Société nationale d'acclimatation de France au Jardin des plantes. II avait épousé en 1830 Louise Blacque (1808-1855), la fille d'un banquier du Finistère. De leur union devaient naître trois enfants, dont la mère de Louise Poincaré .

Telles sont donc les origines familiales de la future épouse de Poincaré, descendante d'une famille de savants et de magistrats. Tout semble indiquer qu'elle passe toute son enfance dans les jardins du Muséum d'histoire naturelle, ses parents décidant d'habiter dans les logements de fonction du Muséum aux côtés de sa grand-mère.

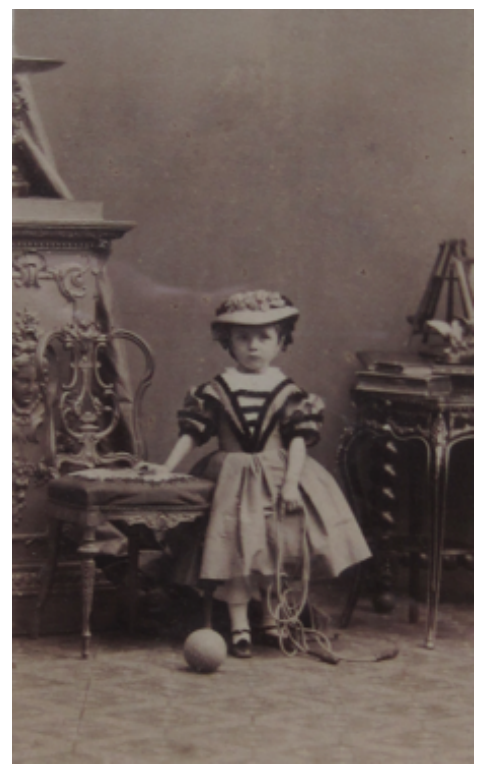

\section{Louise Poulain d'Andecy enfant (Fonds de M. et Madame Poincaré)}

Louise Poulain d'Andecy est la seule fille de la famille. Son frère aîné, Maurice (1853-19-), fera une carrière de chef de service au Crédit foncier, suivant les traces de son père ${ }^{10}$; son second frère ainé Clément Louis Paul (1856-1932) embrassera également une carrière de banquier comme chef de service à la Banque de France; son premier frère cadet, Stéphane (1859-1949) sera quant à lui

\footnotetext{
${ }^{8}$ Archives nationales, dossier d'Henri Poulain d'Andecy, F/1BI/170/23.

${ }^{9}$ Sur la vie et l'œuvre d'Isidore Geoffroy Saint Hilaire : Salf Éric, "Isidore Geoffroy Saint-Hilaire : "Digne de son père" (1805-1861) ", Communication présentée aux séances du 25 novembre 1989 et 27 janvier 1990 de la Société française d'histoire de la médecine. Texte en ligne, consulté le 19 juin 2012 : http://www.biusante.parisdescartes.fr/sfhm/hsm/HSMx1990x024x002/HSMx1990x024x002x0111.pdf. 10 Il obtiendra la Légion d'honneur en 1912: Archives nationales, dossier de Légion d'honneur, LH/19800035/207/27168.
} 
viticulteur dans le Gard et le second Albert Charles Émile (1863-1902) fera sa vie dans le Var, probablement à Hyères, où il décédera assez jeune ${ }^{11}$.

\section{Jean Baptiste Henri Poulain d'Andecy (1818-1884)}

Comme on l'a vu, deux fils d'Henri Poulain d'Andecy suivent le modèle paternel en faisant carrière dans la banque. En 1881, au moment du mariage de sa fille avec Henri Poincaré, il est administrateur du Crédit foncier. Cependant, son parcours de carrière a été bien plus riche et complexe.

Il a fait des études " solides et complètes ${ }^{12}$ dans les collèges royaux de Saint-Louis et de Versailles où il s'est préparé à entrer à l'École polytechnique. Pour une raison inconnue, il ne se présente pas au concours et passe une licence de droit à Paris avant de s'inscrire au Barreau, où il ne semble pas exercer d'activité régulière.

Ses goûts le portent en effet vers l'érudition, les études bibliographiques et la paléographie. Au début des années 1840, Poulain d'Andecy fréquente assidûment la Bibliothèque de l'Arsenal où il entretient des relations suivies avec son conservateur en chef, Charles Cayx (1793-1858) ${ }^{13}$ ainsi qu'avec son conservateur-adjoint Pierre Varin (1802-1849) ${ }^{14}$. Sans qu'on puisse l'affirmer avec certitude - les sources parlent d'une relation d'élève à professeur - il a probablement suivi les enseignements de l'un ou l'autre d'entre eux. Ces activités bibliographiques, menées avec Varin à partir de 1844, lui apportent plus de satisfaction que la profession d'avocat et il songe alors à en faire son métier. C'est dans cet état d'esprit qu'il écrit donc au ministre de l'Instruction publique, AbelFrançois Villemain (1790-1870), le 22 juillet 1844 :

Monsieur le ministre,

J'ai I'honneur de solliciter de vos bontés une place d'employé à la Bibliothèque de l'Arsenal. L’ordonnance Royale du 22 février 1839 crée deux places d'employés surnuméraires pour cette bibliothèque, et aucune n'est actuellement occupée.

Ayant trouvé dans un savant érudit, Conservateur adjoint de cette bibliothèque [probablement Pierre Varin], l'intérêt affectueux d'un professeur pour un ancien élève, je me suis livré sous sa direction à des études bibliographiques et paléographiques et j'ai été initié par lui aux travaux d'érudition vers lesquels m'entraînent mes goûts et mes études antérieures. Le titre modeste

\footnotetext{
${ }^{11}$ Nous tenons ces informations de M. et Mme Poincaré qui nous ont permis de consulter l'arbre généalogique de leur famille ainsi que du site généalogique dédié à la famille Blacque, consulté le 20 juin 2012 : http://gw3.geneanet.org/pblaque?lang=fr\&m=NG\&pz=patrick\%2Bhenri\%2Bbernard\&nz=blaque\&ocz=0\&n=bla cque\&t=N

${ }^{12} C^{\prime}$ est ce que nous indique une lettre dans un dossier conservé aux Archives nationales, F/17/21538/a.

${ }^{13}$ Formé à l'École normale supérieure, Charles Cayx avait commencé sa carrière au sein de la Bibliothèque de l'Arsenal. Il s'agissait au départ d'un emploi d'attente qu'il avait obtenu grâce à la recommandation de son conservateur, Joseph de Treneuil (1766-1818) mais il devait finalement rester associé à cette institution durant toute sa carrière, cumulant ses fonctions de conservateur en chef puis d'administrateur avec ses autres emplois. Professeur agrégé au Lycée Charlemagne durant de nombreuses années, il devait prendre part à la rédaction des premiers programmes et précis historiques en usage à l'Université et occuper ensuite les fonctions d'inspecteur de l'Académie de Paris, de député conservateur et, en fin de carrière, de vice-recteur de la même Académie. Sur la vie et l'œuvre de Charles Cayx, cf. Robert, A., E. Bourloton, et al. (Eds), Dictionnaire des parlementaires français comprenant tous les membres des assemblées françaises et de tous les ministres français depuis le $1^{\text {er }}$ mai 1789 jusqu'au $1^{\text {er }}$ mai 1889, avec leurs noms, état civil, état de service, actes politiques, votes parlementaires, etc. Paris, Bourloton, 1890-1891. Voir également: "Cayx, Charles (17931858), vice-recteur de l'Académie de Paris ", texte en ligne consulté le 21 juin 2012 :

http://pages.textesrares.com/index.php/Rubriques/Cayx-Charles-1793-1858-vice-recteur-de-I-Academie-deParis.html.

${ }^{14}$ Agrégé d'histoire en 1833, Pierre Varrin avait été professeur d'histoire de la Maison des pages du roi Charles $X$. Professeur au collège royal de Reims et archiviste municipal de la ville. Il sera doyen de la Faculté des lettres de Rennes avant d'être nommé en 1844 conservateur-adjoint de la Bibliothèque de l'Arsenal.
} 
que je serais heureux d'obtenir, en me fixant auprès de ce maître éclairé me permettrait de continuer utilement mes travaux et de mériter ainsi les bontés que je réclame de votre Excellence.

Je ne puis me recommander auprès de vous, Monsieur le Ministre, par aucun travail connu, mais j'invoquerai, comme garantie de mon vif désir d'être utile, la considération publique dont jouit ma famille. Je suis proche parent de Messieurs Brière de Mondétour, Inspecteur divisionnaire des Ponts et Chaussées, Brière de Valigny, Conseiller à la Cour de cassation, Isidore Geoffroy Saint Hilaire, inspecteur général des Études, Buchère doyen des notaires de Paris.

Je suis, avec un profond respect, Monsieur le Ministre, le très humble et bien obéissant serviteur. Henri Poulain d'Andecy Rue des grands Augustins $n^{\circ} 18^{15}$.

Cette lettre de candidature est intéressante à plus d'un titre. Elle nous dévoile en premier lieu le riche réseau de relations familiales dont bénéficie Poulain d’Andecy: Alexandre François Brière de Mondétour (1783-1850) est un ancien élève de l'École polytechnique (promotion 1801) et il est alors inspecteur divisionnaire des Ponts-et-Chaussées ${ }^{16}$. Clément François Marie Brière de Valigny (17851872), conseiller à la Cour de cassation, est en fait son frère cadet et a fait changer son patronyme de Mondétour en Valigny. Quant à Isidore Geoffroy Saint Hilaire il n'est autre, comme on l'a vu, que le père de sa future épouse Pauline. Ce faisant, on apprend ainsi qu'Henri Poulain d'Andecy est un parent proche de la famille Geoffroy Saint Hilaire. Enfin, et surtout, ce document nous indique sans ambiguïtés que le futur beau-père de Poincaré est à cette époque dans une situation d'aisance financière qui l'autorise à briguer un modeste poste d'employé et à négliger une carrière bien plus lucrative à laquelle il s'est formé et où il pourrait trouver de nombreux et puissants soutiens.

Ce premier acte de candidature n'est pas suivi d'effet. L'année suivante c'est donc Brière de Valigny qui s'adresse au ministre Narcisse-Achille de Salvandy (1795-1856) :

Ce jeune postulant (il a 27 ans) a fait des études solides et complètes dans les collèges royaux de St Louis à Paris et de Versailles; il a fait son droit à Paris et, depuis plus de trois ans il suit le barreau, mais il s'est occupé surtout de travaux bibliographiques et l'appui qu'il a trouvé auprès de M. Varin lui a été sous ce rapport d'un grand avantage ; c'est dans le but de continuer les travaux qu'il a commencés auprès de ce savant professeur qu'il sollicite sa nomination à la bibliothèque de l'Arsenal. Je puis attester à votre excellence que ce jeune homme est laborieux, instruit et que sa conduite n'a jamais donné lieu à aucun reproche ; je le crois digne sous tous les rapports des bontés que je prie votre excellence de lui accorder ${ }^{17}$.

Quelques jours plus tard, ce sera au tour d'Isidore Geoffroy Saint Hilaire de recommander la nomination de son jeune parent :

Monsieur,

Un de mes parents, M. d'Andecy, qui travaille depuis quelque temps, sans titre, à la bibliothèque de l'Arsenal, vient d'adresser une demande à Monsieur le Ministre, à l'effet d'être nommé surnuméraire. J'ose prier instamment de mettre la demande de M. d'Andecy sous les yeux de Monsieur le Ministre, et de lui être favorable en ce qui dépend de vous.

Je ne crains pas de vous assurer que $M$. d'Andecy sera pour la bibliothèque une excellente acquisition. Je ne connais personne qui aime plus que lui les lettres, la philosophie et le travail. II a acquis une instruction étendue et solide; et c'est par suite de ses penchants littéraires qu'il a renoncé à suivre la carrière du barreau où ses débuts eussent été favorisés par nos liaisons de proche parenté ou d'amitié avec plusieurs magistrats éminents de la Cour de cassation et de la Cour royale. Je ne dois pas oublier d'ajouter que $M$. d'Andecy a poussé assez loin l'étude des sciences, celle des mathématiques en particulier, et qu'il peut rendre ainsi des services très variés à la bibliothèque.

\footnotetext{
${ }^{15}$ Archives nationales, F/17/21538/a.

${ }^{16}$ On peut supposer qu'il est affecté, au moins pendant quelques années, dans la Marne puisqu'on trouve parmi ses rapports publiés un travail consacré aux canaux de la Marne et de la Saône : Note relative au tracé du canal de jonction de la Marne à la Saône, entre Langres et Pontaillier, et considérations en faveur du projet de descendre à la Saône par la vallée de la Vingeanne, Paris : Imprimerie de Schneider et Langrand, 1840.

17 Lettre de Clément Brière de Valigny à Narcisse-Achille de Salvandy, 15 avril 1845, Archives nationales, $\mathrm{F} / 17 / 21538 / \mathrm{a}$.
} 
M. d'Andecy ne demande d'ailleurs qu'un emploi très modeste ; il a de l'aisance ; il peut suivre une carrière lente et peu avantageuse du côté de la fortune, mais dans laquelle ses goûts studieux et littéraires seront pleinement satisfaits.

Veuillez me pardonner, Monsieur, d'insister auprès de vous au sujet de mon jeune parent : je le fais autant par conviction de son mérite que par affection pour lui.

Veuillez agréer, je vous prie, la nouvelle expression de ma haute considération et de mes sentiments dévoués.

I. Geoffroy Saint-Hilaire ${ }^{18}$

Au final, une fois réglé un problème gênant de confusion sur son patronyme - le ministère pensant un moment qu'il y avait deux candidats sur le poste, Henri Poulain et Henri d'Andecy - Henri Poulain d'Andecy sera nommé employé surnuméraire dans cette bibliothèque en janvier 1846 . II y restera environ deux ans.

Les trois journées révolutionnaires des 22, 23 et 24 février 1848 débouchent sur le renversement de la Monarchie de Juillet et sur la proclamation de la République. De quelle manière Poulain d'Andecy prend-il part à ces événements ? Les sources ne l'indiquent pas mais donnent à penser qu'il est un révolutionnaire actif. Ainsi, on le retrouve au printemps 1848 exerçant la fonction de souscommissaire de l'arrondissement d'Ambert, dans le Puy-de-Dôme. La République veut bien-sûr exercer un contrôle strict sur l'ensemble du territoire national et elle a entrepris de nommer des serviteurs fidèles pour administrer les régions et empêcher toute contre-révolution. Les autorités louent alors le goût de Poulain d'Andecy pour la conciliation, dans un contexte local difficile, et ses opinions « franchement et anciennement républicaine » :

M. d'Andecy est âgé de 30 ans. Il avait acquis dans le temps toutes les connaissances nécessaires pour entrer à l'École polytechnique, où il ne s'est pas présenté. Il est licencié en droit et a fait son stage d'avocat à Paris, mais il n'en a jamais exercé la profession. Ami de l'étude, il a pendant plusieurs années, secondé, comme simple amateur, ou avec le titre d'attaché, MM. les conservateurs de la Bibliothèque de l'Arsenal. C'est un homme d'esprit et de conduite ; rigide en toute espèce de probité ; de mœurs douces quoique tenace dans ses principes républicains. On le croit propre à être chargé d'une préfecture ${ }^{19}$.

Il sera finalement nommé sous-préfet d'Ambert le 22 juillet 1848 . Ses débuts dans la fonction semblent satisfaisants, tant pour lui que pour ses supérieurs. Cependant, le décès de son père en octobre 1848, change quelque peu sa situation. Il se retrouve de fait en situation de chef de famille, chargé du devenir de deux sœurs mineures et obligé de veiller à son patrimoine qui est situé dans l'arrondissement d'Épernay. À partir de ce moment il demandera régulièrement à obtenir un poste dans un arrondissement géographique plus proche, sollicitant une fois de plus des soutiens familiaux et amicaux (Alexandre François Brière de Mondétour, Clément Brière de Valigny, etc.). Sans succès.

Ses soucis personnels semblent lui nuire quelque peu. Dans un rapport de septembre 1849, ses supérieurs émettent ainsi plusieurs critiques sur sa situation de famille difficile et sur ses antécédents politiques :

La conduite politique de M. d'Andecy n'est pas bien nette et ne peux pas inspirer une entière confiance.

[...]

M. d'Andecy ne manque ni d'intelligence ni d'activité, et lorsqu'il aura acquis plus de tact et une connaissance plus approfondie des hommes pourra faire un bon sous-préfet; mais il faudrait qu'il fût placé dans un autre arrondissement. À Ambert son origine toute révolutionnaire le met dans une position difficile et dont il ne pourra pas sortir. Domine par une [cadence ?] hostile aux partis modérés et inspire une vive défiance dans ce parti ${ }^{20}$.

Vers la fin du mois d'octobre 1849 de la même année, Henri Poulain d'Andecy est nommé souspréfet de l'arrondissement de Nantua dans l'Ain. Toujours éloigné de sa famille, il persiste toujours dans ses demandes de rapprochement géographique. Toutefois, les rapports le concernant sont

\footnotetext{
18 Lettre d'Isidore Geoffroy Saint-Hilaire au cabinet de Narcisse-Achille Salvandy, 26 avril 1845, Archives nationales, F/17/21538/a.

${ }^{19}$ Archives nationales, $\mathrm{F} / 1 \mathrm{bl} / 170 / 23$.

${ }^{20}$ Rapport sur Henri Poulain d'Andecy, 10 septembre 1849, Archives nationales, F/1bl/170/23.
} 
beaucoup moins critiques puisque ses supérieurs voient en lui un " préfet modèle qui exerce sur son arrondissement une profonde influence morale ${ }^{21}$. En 1850, il perd une de ses deux sœurs et il bénéficie d'une nouvelle recommandation du prince de Canino, descendant de Napoléon Bonaparte (probablement Charles Lucien Bonaparte, 1803-1857). Toujours sans succès. II se marie à l'automne 1851 et, deux jours après le coup d'État de Louis Napoléon-Bonaparte, il démissionne de son poste de sous-préfet au nom de son engagement républicain.

Sa carrière dans la haute administration d'État aura donc duré trois ans. II poursuivra et terminera sa carrière dans le monde de la finance, devenant l'administrateur du Crédit foncier de France.

De l'érudition d'Henri Poulain d'Andecy il reste peu de traces. On connaît de lui une brochure biographique publiée en 1878 sur le médecin Joseph-Alexandre Auzias-Turenne (1812-1870) : AuziasTurenne, sa vie son œuvre $(1878)^{22}$. Celui-ci avait consacré une grande partie de sa vie à des travaux sur l'inoculation préventive de la syphilis, sur le modèle de la variolisation. Ami d'Isidore Geoffroy Saint Hilaire, il était sans nul doute un ami très proche de Poulain d'Andecy, au point de le charger d'exécuter son testament.

On lui connaît un engagement en faveur de la création de l'École alsacienne de Paris, une institution d'éducation créée en en 1873 pour accueillir les élèves des territoires alsaciens. II fera partie de son Conseil d'administration, aux côtés du chimiste Charles Friedel (1832-1899) ou de l'historien Gabriel Monod (1844-1912)

Notons pour finir que plusieurs sources mentionnent qu'il était titulaire de la Légion d'honneur sans qu'il soit possible de retrouver la trace de cette nomination $^{24}$.

\section{Une famille influente: les débuts de carrière d'Henri Poincaré}

La mariage de Poincaré avec Louise Poulain d'Andecy a lieu à Paris en avril 1881. II unit le représentant d'une influente famille lorraine à une jeune fille dont l'entourage dispose de nombreuses entrées dans la société parisienne et dans les cabinets ministériels... dont celui de I'Instruction Publique.

Dès le 15 février 1881, Henri Poulain d'Andecy écrit ainsi à une cousine pour lui annoncer le futur mariage des jeunes gens; dans sa lettre, on apprend qu'il estime beaucoup son futur gendre et qu'il espère bien le voir poursuivre rapidement sa carrière à Paris :

Notre futur gendre n'a pas encore accompli sa $27^{\text {ème }}$ année et est, depuis 15 mois, chargé de cours en mathématiques supérieures à la faculté des sciences de Caen, avec l'espoir d'arriver à Paris dans un temps peu éloigné. Notre fille accepte avec une entière confiance, et une entière satisfaction réfléchie, l'avenir qui lui est offert et nous sommes heureux de l'unir à un homme non moins distingué par ses qualités intimes que par sa valeur scientifique. Nous trouvons en effet en $M$. Henri Poincaré une grande simplicité de manières, une modestie naturelle et un caractère doux et enjoué. II est originaire de Nancy, où son père, médecin honoré par tous, est lui-même professeur à la faculté de médecine de cette ville. II n'a qu'une sœur qui est mariée depuis deux ans à M. Boutroux, maître de conférence de philosophie à l'École Normale Supérieure de Paris et l'union la plus grande règne dans cette famille ${ }^{25}$.

\footnotetext{
${ }^{21}$ Ibidem.

22 Poulain d'Andecy, Henri, Auzias-Turenne, sa vie, son œuvre, Paris, Germer Baillière 1878.

${ }^{23}$ Théodore Beck, Mes souvenirs 1890-1922, Paris, Fischbauer, 1934.

${ }^{24}$ Le faire part de mariage de Poincaré mentionne ce titre.

${ }^{25}$ Cette lettre nous a gracieusement été communiquée par M. François Poincaré. Par ailleurs, sur une lettre envoyée par le mathématicien Georges Henri Halphen (1844-1889), Poincaré avait recopié la lettre dans laquelle Henri Poulain d'Andecy lui annonçait son accord quant à son mariage. Ce fragment, daté du 23 février 1881, était probablement destiné à être envoyé à sa famille : "Mon cher Henri, j'ai reçu ce matin la demande de la main de ma fille que $M$. votre père $m^{\prime}$ adresse en votre nom. Je lui réponds avec empressement - et à
} 
Soucieuse d'accélérer la nomination de son gendre dans la capitale, la famille Poulain d'Andecy sollicitera ainsi les faveurs d'une certaine Madame Kestner en souvenir d'une grand-mère commune ${ }^{26}$. L'épouse d'Henri Poulain d'Andecy, Pauline, écrit ainsi à cette dame, quelques semaines après le mariage :

Madame et vénérée amie,

Nous avions appris par des amis communs vos grandes inquiétudes si rapidement justifiées par un cruel dénouement, et nous aurions voulu vous exprimer de vive voix notre douloureuse sympathie. Deux fois, sans succès, nous avons tenté d'arriver jusqu'à vous, mais nous conservons l'espoir d'être plus heureux dans une nouvelle démarche.

Malgré votre douleur j'ose vous parler de nos préoccupations pour notre jeune ménage et recommander à la sollicitude maternelle que vous voulez bien nous témoigner en souvenir de notre grand-mère, une note destinée à appeler l'attention et la bienveillance du ministre de l'instruction publique et du directeur de l'Enseignement supérieur sur la candidature de Mr Henri Poincaré à l'une des positions de l'enseignement mathématique supérieur à Paris.

Les témoignages que nous recueillons de tous nos amis de l'Académie des Sciences sur le mérite exceptionnel de Mr Henri Poincaré nous garantissent que sa nomination à Paris serait considérée comme une distinction parfaitement justifiée; et c'est avec confiance que nous plaçons sa candidature sous vos auspices.

Mr d'Andecy se joint à moi, Madame et vénérée amie, pour vous renouveler l'expression de notre reconnaissance et celle de notre plus affectueux respect.

$\mathrm{PA}^{27}$

Tout indique que cette vénérée amie appartient à la famille de Charles Kestner (1803-1870). Celui-ci était le fils d'un industriel alsacien, Karl Kestner (1776-1846), qui avait fondé une usine de produits chimiques à Kœnigshoffen et qui en dirigeait une autre à Thann, dans le Haut-Rhin. Son grand-père, Jean-Chrétien Kestner (1741-1800) avait, quant à lui, mené une carrière de diplomate et il avait épousé Charlotte Buff (1753-1828), une ancienne maîtresse de Goethe qui devait être immortalisée dans Les souffrances du jeune Werther (1774). Après des études de droit à Francfort et des études de chimie à Göttingen, Charles Kestner était devenu l'associé de son père dans la fabrique de Thann. Chimiste de grande renommée, il avait découvert l'acide paratartrique ainsi qu'un procédé de fabrication en continu de l'acide sulfurique. Élu conseiller municipal de Thann en 1843, il avait été également député du Haut-Rhin à l'Assemblée constituante de 1848. Par la suite, son hostilité à l'Empire et ses prises de position républicaines lui avaient valu la prison et l'exil et l'avaient obligé à quitter la sphère publique. II avait épousé en 1827, Marie Jeanne Rigau (1806-1890), fille du baron d'Empire Antoine Rigau (1758-1820), qui avait transformé très vite son salon en foyer antibonapartiste.

vous en même temps - que nous sommes heureux et fiers de vous voir entrer dans notre famille. Ma fille accepte avec une entière confiance et avec la satisfaction la moins dissimulée l'avenir que vous lui offrez, et j'aime à croire que de votre côté vous apprécierez de plus en plus les qualités sérieuses de celle que vous choisissez pour compagne ".

${ }^{26}$ On peut supposer que cette Madame Kestner était une amie très proche de la grand-mère de l'épouse d'Henri Poulain d'Andecy.

${ }^{27}$ Voici le texte de la note pour le ministre qui accompagne cette lettre: "Des nominations de maîtres de conférences à l'École Normale Supérieure et de suppléants à la Faculté des Sciences de Paris, pour le haut enseignement mathématique, doivent avoir lieu au mois d'octobre 1881. Mr Henri Poincaré, ingénieur des mines, docteur es sciences, chargé du cours de calcul différentiel et intégral à la Faculté des Sciences de Caen est un des candidats. Une mention très honorable lui a été décernée en séance publique le 14 mars 1881, dans le concours pour le grand prix des sciences mathématiques et ses importants travaux, justement appréciés en France et à l'étranger lui ont valu l'honneur d'une présentation par la section de géométrie de l'Académie des Sciences, lors de l'élection récente pour le remplacement de $M^{r}$ Chasles. $M^{r}$ Henri Poincaré a épousé au mois d'avril 1881 mademoiselle d'Andecy, petite fille d'Isidore Geoffroy $S^{t}$ Hilaire ». Cette note, qui provient de la correspondance conservée par la famille Poincaré, est également présente dans son dossier de carrière : Archives nationales, F/17/23168. La lecture de ce dossier permet de voir que Poincaré sera candidat sur un poste de maître de conférences à l'École normale supérieure pour lequel on lui préférera Jules Tannery (18481910). 
De cette union naîtront cinq filles, qui toutes épouseront des hommes politiques d'envergure nationale. Hortense Kestner (1840-1913) se mariera ainsi avec Charles Floquet (1828-1896), avocat et président du Conseil en 1888 ; Fanny Kestner (1831-1850) épousera Victor Chauffour (1819-1889), professeur de droit à Strasbourg, conseiller d'État et représentant du Haut-Rhin à l'Assemblée Nationale ; Mathilde Kestner (1832-1916) s'unira quant à elle à Jean-Baptiste Adolphe Charras (18101865), sous-secrétaire d'État au ministère de la Guerre en 1848 , et chef d'État-Major du général Cavaignac ; Céline Kestner (1838-1894) épousera Auguste Scheurer (1833-1899), président du Sénat qui fera éclater au grand jour l'innocence du capitaine Dreyfus ${ }^{28}$; enfin, Eugénie Kestner (1828-1862) se mariera avec l'industriel Camille Risler (1821-1881) futur maire du septième arrondissement de Paris, dont la fille, Mathilde Eugénie Risler (1850-1920), épousera Jules Ferry en 1875... ${ }^{29}$

À la lumière de ces informations biographiques, qui illustrent l'importance de la famille Kestner sur la scène politique française, on peut supposer que la famille Poulain d'Andecy destine sa lettre, soit à la veuve de Charles Kestner, soit à l'une de ses filles encore en vie en 1881. La réponse à cette requête ne se fera pas attendre. Elle s'adresse à Pauline Poulain d'Andecy.

Chère Madame et amie,

Votre lettre m'est arrivée à Rueil, où je suis venue pour me remettre au milieu des soins et de l'affection de mes enfants, de la terrible secousse qui m'a enlevé l'un d'eux [peut-être Camille Risler?]. J'ai bien souffert mais c'est dans l'oubli de soi-même que réside la meilleure consolation; et en voyant le bonheur des autres, on souffre moins de sentir sa part diminuée.

Je viens d'écrire à $M$. Ferry en lui envoyant la note que vous avez jointe à votre lettre. Je ne fais aucun doute que les titres de M. Poincaré justifient assez sa demande pour qu'il doive, à eux seuls, la réussite que j'espère. Je vous remercie, chère Madame et amie, d'avoir assez compté sur ma vieille amitié pour $m$ 'employer à vous rendre ce léger service (en ce qui me concerne du moins) et je me féliciterai avec vous du but atteint quand je saurai votre chère fille rapprochée de vous.

Je suis ici jusqu'à la fin du mois et quitterai d'ici pour me rendre en Alsace. Croyez à tout le plaisir que nous aurions, ma fille et moi, si la visite que vous et Mr d'Andecy me promettez pouvait s'étendre jusqu'à Rueil 31 rue de Versailles.

J'aurai soin de vous adresser la réponse de $M$. Ferry à moins qu'elle ne vous arrive par voie administrative, avant celle que je pourrai vous donner. En attendant et, quoi qu'il arrive, croyezmoi toujours votre dévouée et maternelle amie

Kestner

Un souvenir particulièrement aimable à $M$. $d^{\prime}$ Andecy et à votre frère ${ }^{30}$.

Le 19 octobre 1881, Poincaré sera finalement nommé maître de conférences d'analyse à la Faculté des Sciences de Paris. Cette intervention auprès de la famille Kestner n'aura fait probablement qu'accélérer une nomination certaine mais on peut gager que sans elle le parcours de Poincaré aurait suivi la voie normale des nominations dans de petites facultés de province avant la promotion parisienne ${ }^{31}$. Ajoutons par ailleurs que la famille Kestner ne sera pas le seul soutien de Poincaré au ministère de l'Instruction publique à cette époque : I'historien Alfred Rambaud (1842-1905), un ami très proche de la famille Poincaré, y est le directeur de cabinet de Jules Ferry ${ }^{32}$.

\footnotetext{
${ }^{28}$ Comme le voulait une tradition protestante en vigueur à l'époque, il avait accolé le nom de sa femme au sien et se fit ainsi appeler Auguste Scheurer-Kestner.

29 Pour plus de détails sur famille Kestner, voir Roumieux André, "Les déchirements d'un républicain alsacien ", dans Scheurer-Kestner, Auguste, Mémoires d'un sénateur dreyfusard, Strasbourg, Bueb et Remaux, 1988. On se reportera également au Dictionnaire de biographie française, tome XVIII.

${ }^{30}$ Lettre conservée aux Archives Poincaré, à Nancy.

${ }^{31}$ Rappelons que les traitements des professeurs de la Sorbonne sont à cette époque supérieurs à ceux de leurs homologues en poste en province.

${ }^{32}$ Sur les relations entre les familles Rambaud et Poincaré, voir Boutroux Aline, Vingt ans de ma vie... Op. cit.
} 


\section{Épilogue}

Bien qu'il ne nous apprenne que peu choses sur Louise Poincaré et sur les circonstances de sa rencontre avec Poincaré, le récit de la trajectoire d'Henri Poulain d'Andecy nous donne des informations importantes sur le milieu social dans lequel le mathématicien a pu évoluer au cours de sa vie. Faut-il voir dans cette union le résultat de la stratégie familiale d'un jeune homme ambitieux (et relativement courante chez les polytechniciens) ? Nous laisserons cette question sans réponse.

Henri et Louise Poincaré auront quatre enfants, un fils et trois filles, qui toutes feront de très bonnes études secondaires mais pas d'études supérieures. Leur première fille, Jeanne (1887-1974), sera un temps la secrétaire de son père avant d'épouser Léon Daum (1887-1966) en 1913. Ingénieur des mines, héritier de la célèbre famille de cristalliers nancéiens, il sera le premier représentant de la France auprès de la Communauté économique du charbon et de l'acier (CECA). Yvonne (1889-1939), la seconde, de santé très fragile, restera célibataire et passera toute sa vie auprès de sa mère puis s'installera chez les Daum. Leur troisième fille, Henriette (1890-1970) épousera un ingénieur civil employé dans les mines de la Sarre (où Daum avait commencé sa carrière) puis reconverti dans l'élevage des poules à Talloires. Lassée de sa condition, elle divorcera vers 1945 pour s'installer elle aussi chez les Daum. Enfin, Léon Poincaré (1893-1972), suivra les traces de son père à l'École polytechnique puis à l'École des mines et fera une brillante carrière d'ingénieur général de l'air. II épousera Emma Motte (1895-1983) ${ }^{33}$.

Les traces laissées par Louise Poincaré sont très peu nombreuses. Quelques photographies de famille sur lesquelles elle apparaît souvent en costume de deuil, six lettres de son mari datant probablement des années 1885-1886 dans lesquelles il raconte ses visites de candidature à l'Académie des sciences $^{34}$ et quelques lettres postérieures à la mort de celui-ci concernant la postérité de ses travaux ou la célébration de sa mémoire ${ }^{35}$.

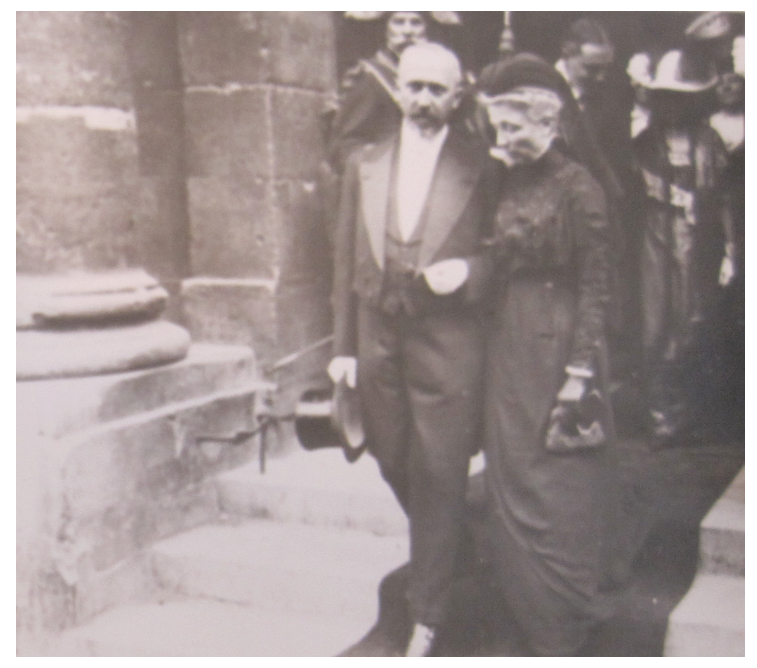

Louise Poincaré en 1913 au mariage de sa fille Jeanne (Fonds de M. et Mme Poincaré)

À la mort de son mari en 1912, elle s'installera dans leur maison familiale de Lozère, près de Palaiseau. Cette meulière avait été achetée par Eugénie Poincaré, sa belle-mère, en 1893. Après le

\footnotetext{
${ }^{33}$ Nous tenons ces informations de M. François Poincaré et de Madame Karin Blanc.

34 Elles sont consultables sur le site de la correspondance d'Henri Poincaré : http://www.univnancy2.fr/poincare/chp/.

${ }^{35}$ Voir ainsi les échanges des descendants de Poincaré en 1919 concernant la pertinence de la publication d'un volume 5 des œuvres philosophiques de Poincaré proposé par Louis Rougier: Poincaré Henri, L'opportunisme scientifique / Scientific Opportunism, An Anthology Compiled by Louis Rougier, edited by Laurent Rollet, Berlin, Birkhäuser, 2002.
} 
décès de son mari, Émile Léon Poincaré (1828-1892), elle avait souhaité s'installer en région parisienne à proximité de sa famille. Après sa mort en 1897, cette maison était devenue le lieu de villégiature d'Henri Poincaré et de sa famille. Louise Poincaré survivra 22 ans à son mari. Elle mourra en 1934.

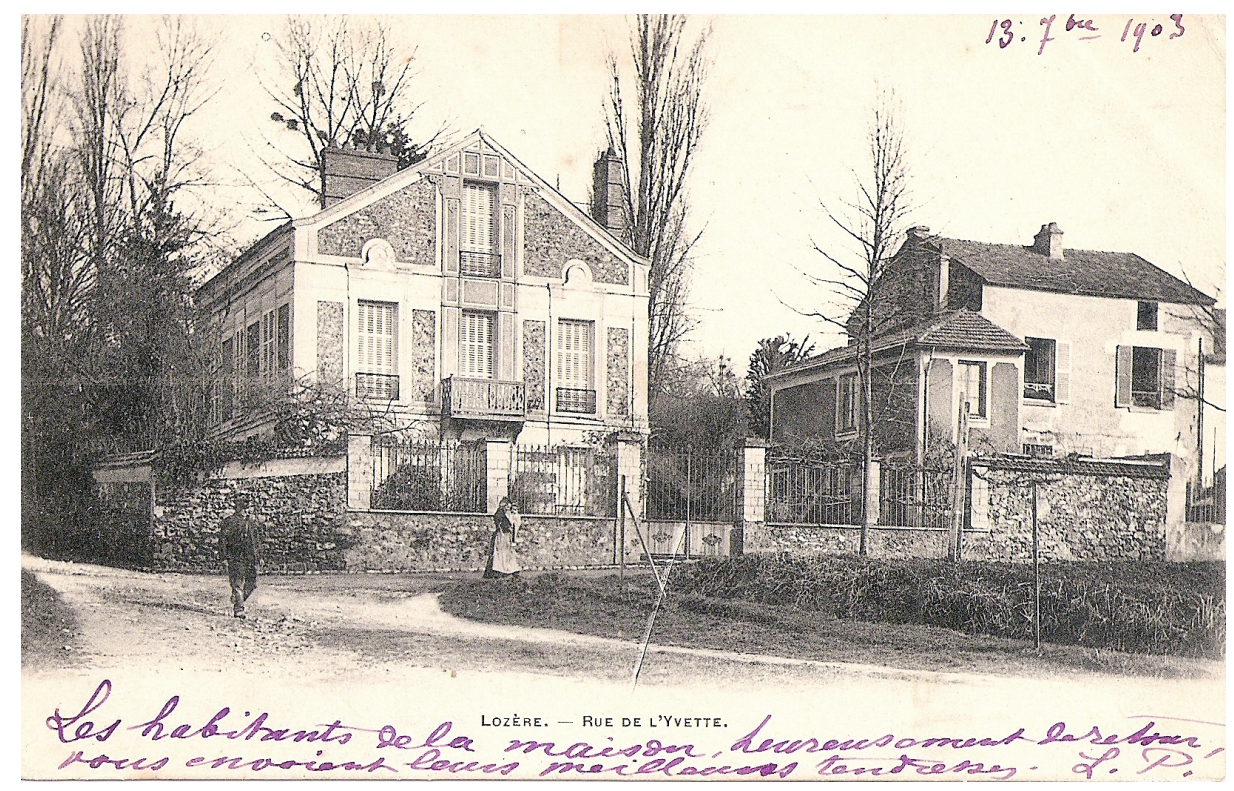

Recto d'une carte postale de la maison de Lozère adressée en 1903 par Louise Poincaré à l'épouse de son frère, Maurice d'Andecy ${ }^{36}$

On ne dispose pas pour l'heure d'une biographie complète du savant. Complète, non pas au sens de définitive, mais au sens de globale. Personne en effet ne semble avoir relevé le défi d'élaborer une biographie qui prendrait en compte l'ensemble de la trajectoire scientifique et privée du savant, qui intégrerait ces épisodes de vie, de carrière, de travail scientifique, d'engagement philosophique etc. dans un portrait général, dont l'enjeu ne serait pas seulement de mieux comprendre les identités multiples du savant mais aussi d'apporter une contribution à l'histoire des 'élites' scientifiques sous la Troisième République. Le récit de cette union matrimoniale ne peut que s'inscrire dans le cadre d'un tel projet biographique.

\footnotetext{
${ }^{36}$ Nous remercions M. Hervé Martin d'avoir mis ce document à notre disposition.
} 